\title{
Optical hollow-core waves in nonlinear Epsilon-Near-Zero metamaterials
}

\author{
C. Rizza, $, 2,2$ A. Ciattoni, ${ }^{2}$ and E. Palange ${ }^{1}$ \\ ${ }^{1}$ Dipartimento di Ingegneria Elettrica e dell'Informazione, Università dell'Aquila, 67100 L'Aquila, Italy \\ ${ }^{2}$ Consiglio Nazionale delle Ricerche, CNR-SPIN 67100 L'Aquila, \\ Italy and Dipartimento di Fisica, Università dell'Aquila, 67100 L'Aquila, Italy
}

(Dated: November 22, 2018)

\begin{abstract}
We investigate non-diffracting hollow-core nonlinear optical waves propagating in a layered nanoscaled metal-dielectric structure characterized by a very small average linear dielectric permittivity (Nonlinear Epsilon-Near-Zero metamaterial). We analytically show that hollow-core waves have a power flow exactly vanishing at a central region and exhibiting a sharp sloped profile at the edges of the regions surrounding the core. Physically, the absence of power flow at the core region is due to the vanishing of the transverse component of the electric displacement field, condition that can be satisfied by the full compensation between the nonlinear and linear dielectric contribution.
\end{abstract}

\section{INTRODUCTION}

Electromagnetic metamaterials have recently attracted significant research attention due to their unique properties generally not available in standard media. The studies of linear metamaterials have opened new possibilities to achieve remarkable effects like as, for example, superlensing [1], optical cloaking [2],nano-photonic circuits [3, 4]. In the same way, the research on the nonlinear metamaterials can overcome the limits of the naturally-occurring materials and can expand the range of possible applications [5 9]. As a leading example, the artificial fabrication of nonlinear materials suggested the way to enhanced the nonlinearity at low intensity. Pendry et al., in their pioneering work [5], proposed a left-handed metamaterials characterized by a huge nonlinear response arising from the concentration (due to microscopic inhomogeneity) of the electromagnetic field within the nonlinear constituents. On the other hand, a simple strategy for making available the nonlinearity is to reduce the linear polarization. Following this strategy, Ciattoni et al. 10] suggested a simple composite structure to achieve very small value of dielectric permittivity where the nonlinear contribution to the dielectric response is not a mere perturbation of the linear one (extreme nonlinear regime). Authors considered a nanoscaled layered structure consisting of alternating metal and dielectric slabs and, in the effective medium scheme, characterized by effective constitutive relations formally coinciding with those of a standard Kerr medium and with response parameters assuming values not available in standard media (nonlinear metamaterials). In these kind of metamaterials, transverse magnetic nonlinear guided waves show a very rich and exotic phenomenology such as the transverse power flow reversing (i.e. the power flow of the considered waves changes its sign along the wave transverse profile, see also Ref.[11]).

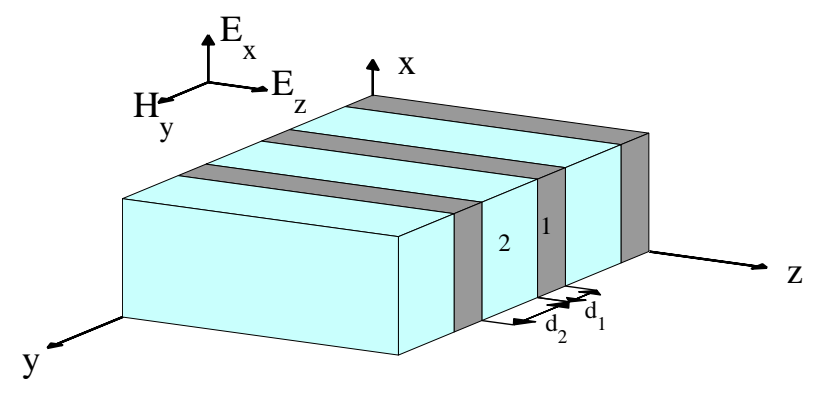

FIG. 1: Geometry of nanoscaled periodic structure and TM electromagnetic field configuration where the unit cell is composed by two layers $(N=2)$.

*Electronic address: carlo.rizza@aquila.infn.it 
In this paper, we consider specific transverse magnetic nonlinear guided waves propagating in a nonlinear EpsilonNear-Zero metamaterial and characterized by a power flow exactly vanishing in a core region and exhibiting a sharp sloped profile at the edges of the side regions surrounding the core. The very marked steepening of the power flow and of the underlying electric field transverse component $E_{x}$ is a consequence of the vanishing of the transverse component of the displacement field along the transverse wave profile, i.e. $D_{x}(x)=0$. Since $D_{x}$ is nonlinearly related to $E_{x}$ and $E_{z}$, this requirement is differently fulfilled within the core (where $E_{x}=0$ ) and the lateral regions (where the effective nonlinear dielectric permittivity vanishes) thus yielding a discontinuity of the transverse field derivatives at the core edges. Such an extreme electromagnetic configuration has, as a main underlying physical mechanism, the exact compensation between linear and nonlinear contributions to the dielectric response in the regions surrounding the core and this is possible since the considered medium can support the extreme nonlinear regime.

\section{NONLINEAR EPSILON-NEAR-ZERO METAMATERIALS}

Consider a monochromatic Transverse Magnetic (TM) field (of time dependence $\exp (-i \omega t)) \mathbf{E}=E_{x}(x, z) \hat{\mathbf{e}}_{x}+$ $E_{z}(x, z) \hat{\mathbf{e}}_{z}, \mathbf{H}=H_{y}(x, z) \hat{\mathbf{e}}_{y}$, propagating along the $z$-axis in a periodic layered medium. The unit cell (of spatial period $d$ ) is composed by $N$ layers filled by different media of thickness $d_{j}$ (as reported in Fig.1 for the case $N=2$ ). We suppose that the $j$-th layer is characterized by the constitutive relation

$$
\mathbf{D}_{\mathbf{j}}=\epsilon_{0} \epsilon_{j} \mathbf{E}_{j}+\epsilon_{0} \chi_{j}\left[\left|\mathbf{E}_{j}\right|^{2} \mathbf{E}_{j}+\gamma_{j}\left(\mathbf{E}_{j} \cdot \mathbf{E}_{j}\right) \mathbf{E}_{j}^{*}\right]
$$

where $\mathbf{E}_{j}, \mathbf{D}_{j}$ are the local electric and electric displacement field, respectively; whereas $\epsilon_{j}$ is the dielectric permittivity of the $j$ th layer and $\chi_{j}$ and $\gamma_{j}$ are the standard nonlinear Kerr parameters. Under the assumption that the light wavelength $\lambda$ is much greater than the spatial period $d$, the homogenized medium behaves like a Kerr medium whose effective constitutive relation is

$$
\mathbf{D}=\epsilon_{0} \epsilon \mathbf{E}+\epsilon_{0} \chi\left[|\mathbf{E}|^{2} \mathbf{E}+\gamma(\mathbf{E} \cdot \mathbf{E}) \mathbf{E}^{*}\right]
$$

where $\mathbf{E}=\left\langle\mathbf{E}_{j}\right\rangle, \mathbf{D}=\left\langle\mathbf{D}_{j}\right\rangle, \epsilon=\left\langle\epsilon_{j}\right\rangle, \chi=\left\langle\chi_{j}\right\rangle$ and $\gamma=\left\langle\chi_{j} \gamma_{j}\right\rangle /\left\langle\chi_{j}\right\rangle$ (each field and parameter is obtained by averaging along the $y$-axis the layer local quantities over the period $d$ ) [10]. All effective parameters appearing in the relation (2) can be tailored by choosing the underlying constituents; for example the averaging of the dielectric permittivity can be used to obtain an efficient loss management (using gain and loss media as underlying layers) [12] and/or an epsilon-near-zero metamaterials (exploiting negative and positive dielectric layers) [4]. It is worth noting that, the nonlinear parameter $\gamma$ can span the whole range of the real values whereas, in the standard Kerr material, it can assume only three values [13] depending on the physical mechanism providing the Kerr nonlinear response.

\section{HOLLOW-CORE WAVES}

We focus here on a situation where $0<\epsilon \ll 1, \chi<0$ and $\gamma<-1$. Eliminating the magnetic field from Maxwell's equations $\nabla \times \mathbf{E}=i \omega \mu_{0} \mathbf{H}, \nabla \times \mathbf{H}=-i \omega \mathbf{D}$ and considering $\mathrm{TM}$ non-diffracting fields of the form $E_{x}(\xi, \zeta)=$ $\sqrt{\epsilon /|\chi|} u_{x}(\xi) \exp (i \beta \zeta), E_{z}(\xi, \zeta)=i \sqrt{\epsilon /|\chi|} u_{z}(\xi) \exp (i \beta \zeta)$, we obtain the system

$$
\begin{aligned}
& -\beta \frac{d u_{z}}{d \xi}+\left[\beta^{2}-\epsilon_{x}^{(N L)}\right] u_{x}=0 \\
& \beta \frac{d u_{x}}{d \xi}-\frac{d^{2} u_{z}}{d \xi^{2}}-\epsilon_{z}^{(N L)} u_{z}=0
\end{aligned}
$$

where $\beta$ is a real propagation constant, $u_{x}$ and $u_{z}$ are real field profiles, $\xi=\sqrt{\epsilon} k_{0} x, \zeta=\sqrt{\epsilon} k_{0} z$ are dimensionless variables and we defined

$$
\begin{aligned}
\epsilon_{x}^{(N L)} & =1-\left[(1+\gamma) u_{x}^{2}+(1-\gamma) u_{z}^{2}\right] \\
\epsilon_{z}^{(N L)} & =1-\left[(1-\gamma) u_{x}^{2}+(1+\gamma) u_{z}^{2}\right] .
\end{aligned}
$$

as effective normalized nonlinear dielectric permittivities. The system of Eqs.(3) is integrable since it admits the first integral $F=\beta^{2} u_{x}^{2}-\left[\frac{d u_{z}}{d \xi}\right]^{2}-\left(u_{x}^{2}+u_{z}^{2}\right)+\left[(1-\gamma) u_{x}^{2} u_{z}^{2}+\frac{1}{2}(1+\gamma)\left(u_{x}^{4}+u_{z}^{4}\right)\right]$, i.e. $d F / d \xi=0$ is satisfied for any solution $u_{x}(\xi), u_{z}(\xi)$ of Eqs.(3). We focus on solutions where $u_{x}$ is spatially even $\left(u_{x}(\xi)=u_{x}(-\xi)\right)$ and $u_{z}$ is odd $\left(u_{z}(\xi)=-u_{z}(-\xi)\right)$ so that we assume the boundary conditions $u_{x}( \pm \infty)=u_{x \infty}$ and $u_{z}( \pm \infty)= \pm u_{z \infty}$. Since $u_{x}$ and 
$u_{z}$ asymptotically approach to two constant values, we require their derivatives to asymptotically vanish and, as a consequence, we obtain from Eqs.(3) (evaluated for $\xi \rightarrow+\infty$ )

$$
\begin{aligned}
\beta^{2} & =2 \gamma\left(1-2 u_{x \infty}^{2}\right) /(1+\gamma) \\
u_{z \infty}^{2} & =\left[1-(1-\gamma) u_{x \infty}^{2}\right] /(1+\gamma) .
\end{aligned}
$$

Substituting this expression of $\beta$ and $d u_{z} / d \xi$, derived from first of Eq.(3), into the expression of $F$, we obtain $F$ as a function of $u_{x}$ and $u_{z}$, so that the nonlinear guided waves correspond to curves of constant $F\left(u_{x}, u_{z}\right)$, in the plane $\left(u_{x}, u_{z}\right)$, which are heteroclinic orbits joining the two saddle points $\left(u_{x \infty}, \pm u_{z \infty}\right)$. It can be shown that such nonlinear guided waves exist if $1 /(1-\gamma)<u_{x \infty}^{2}<1 / 2$ (see Ref. [10] for a more detailed discussion). It is worth noting that Eqs.(3) can be casted into the form

$$
\begin{aligned}
& \beta \frac{d u_{z}}{d \xi}=\left[\beta^{2}-\epsilon_{x}^{(N L)}\right] u_{x} \\
& \beta \frac{d u_{x}}{d \xi}=\frac{\beta^{2} \epsilon_{z}^{(N L)} u_{z}+\left(\epsilon_{x}^{(N L)}-\beta^{2}\right) \frac{\partial \epsilon_{x}^{(N L)}}{\partial u_{z}} u_{x}^{2}}{\epsilon_{x}^{(N L)}+\frac{\partial \epsilon_{x}^{(N L)}}{\partial u_{x}} u_{x}},
\end{aligned}
$$

which is a system of the first order fully equivalent to Maxwell's equations with the two constraints

$$
\beta \neq 0, \quad \epsilon_{x}^{(N L)}+\frac{\partial \epsilon_{x}^{(N L)}}{\partial u_{x}} u_{x} \neq 0 .
$$

We have evaluated the profiles of $u_{x}(\xi)$ and $u_{z}(\xi)$ by solving Eqs.(6) (with a standard numerical Runge-Kutta method) along with the initial conditions $u_{x}(0)=u_{x 0}$ (obtained from the first integral) and $u_{z}(0)=0$. In Fig.(2) we report the profiles of $u_{x}(\xi)$ and $u_{z}(\xi)$ of various nonlinear guided waves (solid lines) and it is evident that, for $u_{x \infty}^{2} \rightarrow 1 / 2$, the profile of $u_{x}(\xi)$ approaches a limiting shape (reported in dotted line) characterized by a core region where $u_{x}=0$ and lateral regions at whose edges $u_{x}$ exhibits very large slopes.
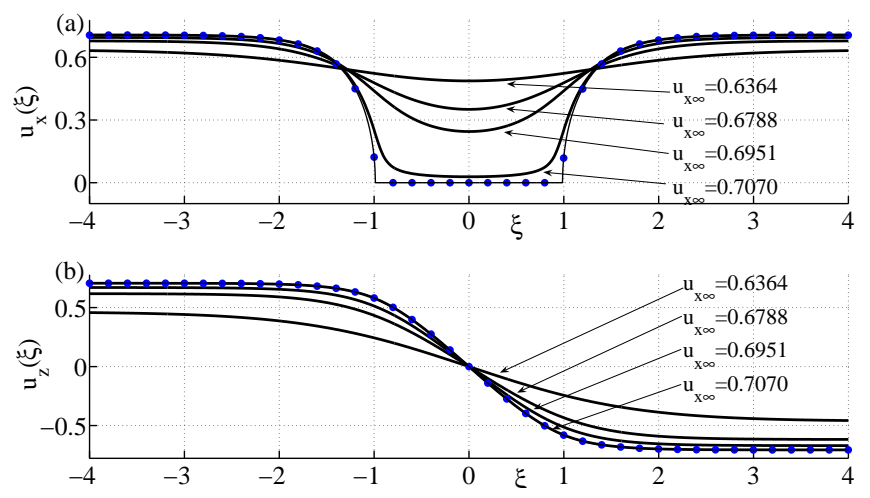

FIG. 2: (Color online) Profiles of $u_{x}(\xi)$ (a) and $u_{z}(\xi)$ (b) of various nonlinear guided waves for $\gamma=-2$ and $u_{x \infty}=$ $0.6364,0.6788,0.6951,0.7070$ (solid lines). The asymptotical $\left(u_{x \infty} \rightarrow \sqrt{1 / 2}\right)$ profiles are also reported (dotted line).

In order to describe the formation of the hollow core wave (reported in Fig.2) we consider the case $u_{x \infty}^{2}=1 / 2$ where $\beta=0$ (see the first of Eqs.(50). In the considered case, the first constraint of Eqs.(77) is violated so that the analysis of this limiting solutions has to be based on Eqs.(3). For $\beta=0$, the first of Eqs.(3) reduces to $\epsilon_{x}^{(N L)} u_{x}=0$ so that $\epsilon_{x}^{(N L)}=0$ or $u_{x}=0$ are the only two possibilities allowed at each $\xi$. Correspondingly, the second of Eqs. (3) (for $\beta=0$ ), if $\epsilon_{x}^{(N L)}=0$, yields

$$
\frac{d^{2} u_{z}}{d \xi^{2}}+\frac{2 \gamma}{1+\gamma}\left(1-2 u_{z}^{2}\right) u_{z}=0
$$

where use has been made of the relation (see the first of Eqs.(4))

$$
u_{x}(\xi)=\sqrt{\frac{1-(1-\gamma) u_{z}^{2}}{1+\gamma}}
$$


if $u_{z}^{2}>1 /(1-\gamma)$ (assuring the reality of $u_{x}$ since here $\left.\gamma<-1\right)$. On the other hand, if $u_{x}=0$, the second of Eqs.(3) yields

$$
\frac{d^{2} u_{z}}{d \xi^{2}}+\left[1-(1+\gamma) u_{z}^{2}\right] u_{z}=0
$$

Since asymptotically $u_{x \infty} \neq 0$, the wave has to be asymptotically characterized by the condition $\epsilon_{x}^{(N L)}=0$ so that, for positive and large $\xi, u_{z}$ satisfies Eq.(8) whose solution (fulfilling the asymptotical requirements $u_{z}(+\infty)=-\sqrt{1 / 2}$ and $\left.\left[d u_{z} / d \xi\right]_{\xi=+\infty}=0\right)$ is

$$
u_{z}(\xi)=-\sqrt{\frac{1}{2}} \tanh \left[\sqrt{\frac{\gamma}{1+\gamma}}\left(\xi-\xi_{0}\right)\right]
$$

where $\xi_{0}$ is a real constant. Since $u_{z}$ of Eq.(11) monotonically decreases as $\xi$ decreases, it is evident from Eq.(9) that

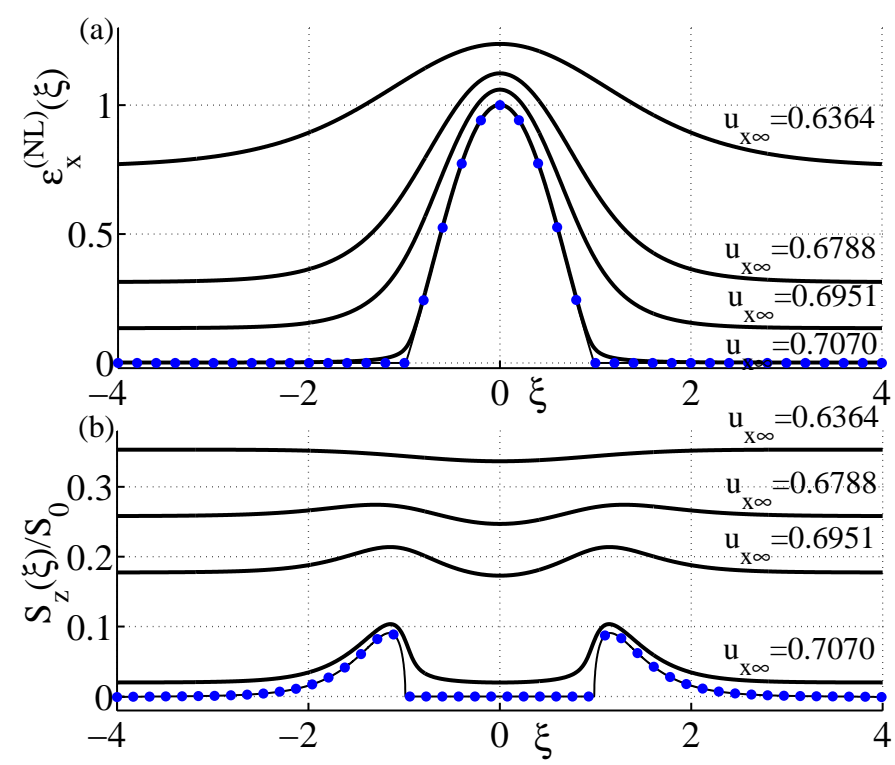

FIG. 3: (a) (Color online) Profiles of the normalized effective nonlinear dielectric permittivity $\epsilon_{x}^{(N L)}(\xi)$ supporting the nonlinear guided waves reported in Fig.2. (b) (Color online) Profiles of the normalized Poynting vector $S_{z}(\xi) / S_{0}$ associated with the nonlinear guided waves reported in Fig.1.

also $u_{x}$ decreases as $\xi$ decreases and consequently a point $\xi=\Delta$ exists at which $u_{x}(\Delta)=0$. For $\xi<\Delta$, the profile of $u_{z}$ of Eq. (11) would yield a complex $u_{x}$ profile through Eq. (9) and this is incompatible with the present discussion. Therefore for $\xi<\Delta$, the condition $\epsilon_{x}^{(N L)}=0$ can not consistently hold so that, in this region, the condition $u_{x}=0$ is the only one left and the field $u_{z}$ is locally the solution of Eq.(10) continuously joined (together with its derivative) with Eq.11 at $\xi=\Delta$. We have numerically evaluated the profile of $u_{z}$ and $u_{x}$ in the above discussed case $\beta=0$ and they are reported in Fig.2 (dotted lines) from which it is evident that the limiting case accurately describes the nonlinear guided waves for $u_{x \infty}>0.7070$ (except for a small region near $\xi= \pm \Delta$ ).

From a physical point of view, the fundamental relation $\epsilon_{x}^{(N L)} u_{x}=0$ characterizing the above discussed hollowcore waves amounts to the vanishing of the transverse component of the displacement field, i.e. $D_{x}=0$, a physical condition differently attained in the core region (where $E_{x}=0$ ) and at the lateral sides $\left(\right.$ where $\left.\epsilon_{x}^{(N L)}=0\right)$ of the hollow-core wave, as discussed above. In Fig.3(a), we plot the profiles of $\epsilon_{x}^{(N L)}$ associated with the nonlinear guided waves reported in Fig. 2 from which we note that, the closer $u_{x \infty}^{2}$ to $1 / 2$, the closer $\epsilon_{x}^{(N L)}$ to zero in the external regions (i.e. $-\infty<\xi<-\Delta$ and $\Delta<\xi<+\infty$ ), thus proving the above discussed mechanism supporting the hollow-core wave. It is worth noting that the relation $\epsilon_{x}^{(N L)}=0$ can be satisfied only if $\left|E_{x}\right| \sim \sqrt{\epsilon /|\chi|}$ and $\left|E_{z}\right| \sim \sqrt{\epsilon /|\chi|}$, so that the occurrence of discontinuities in the field spatial derivatives is a signature of the extreme nonlinear regime where the nonlinear term appearing in the first of Eqs.(2) is comparable with $\epsilon_{0} \epsilon \mathbf{E}$, the linear part of the displacement field. The time averaged Poynting vector $\mathbf{S}=(1 / 2) R e\left[\mathbf{E} \times \mathbf{H}^{*}\right]$, for the considered fields, is given by $\mathbf{S}=S_{0}\left(\beta-d u_{z} / d \xi\right) u_{x} \hat{\mathbf{e}}_{z}$, where $S_{0}=\sqrt{\left(\epsilon_{0} \epsilon^{3}\right) /\left(4 \mu_{0}|\chi|^{2}\right)}$ and we report, in Fig.3(b), the profiles of $S_{z}(\xi) / S_{0}$ corresponding to the nonlinear 
guided waves plotted in Fig.2. Note that, in the limiting case $(\beta=0$, plotted in dotted line in Fig.3(b)), the energy flow exactly vanishes in the core region $-\Delta<\xi<\Delta$ and it shows very steep slopes at $\xi= \pm \Delta$ (following the behavior of the $x$-component of the electric field). In addition the power flow is transversally confined since $S_{z} \rightarrow 0$ for $\xi \rightarrow \pm \infty$ as a consequence of the fact that, asymptotically, $d u_{z} / d \xi \rightarrow 0$.

\section{CONCLUSION}

In conclusion, we have investigated the formation of optical hollow-core waves in Epsilon-Near-Zero metamaterials. The existence of hollow-core waves characterized by a power flow exactly vanishing at a central region is the signature of the extreme nonlinear regime where the linear and nonlinear contributions to the overall medium dielectric response are comparable.

[1] J. B. Pendry, Phys. Rev. Lett. 85, 3966 (2000).

[2] J. B. Pendry, D. Schurig and D. R. Smith, Science 312, 1780 (2006).

[3] N. Engheta, Science 317, 1698 (2007).

[4] M. Silveirinha, N. Engheta, Phys. Rev. Lett. 97, 157403 (2006).

[5] J.B. Pendry, A.J. Holden, D.J. Robbins and W.J. Stewart, IEEE Transaction on microwave theory and techniques, 47, 2075 (1999).

[6] A. A. Zharov, N. A. Zharova, I. V. Shadrivov, Y. S. Kivshar, Applied Physics Letters 87, 091104 (2005).

[7] Y. Liu, G. Bartal, D. A. Genov, X. Zhang, Phys. Rev. Lett. 99, 153901 (2007).

[8] D. A. Powell, A. Alu, B. Edwards, A. Vakil, Y. S. Kivshar, N. Engheta, Phys. Rev. B 79, 245135 (2009).

[9] A. Ciattoni, C. Rizza and E. Palange, Opt. Lett. 35, 2130 (2010).

[10] A. Ciattoni, C. Rizza and E. Palange, Phys. Rev. A 81, 043839 (2010).

[11] A. Ciattoni, C. Rizza and E. Palange, Optics Express 18, 11911 (2010).

[12] S. A. Ramakrishna and J. B. Pendry,Phys. Rev. B 67, 201101(R) (2003).

[13] R. W. Boyd, Nonlinear Optics (Academic Press, New York, 1994). 